J. Klin. Endokrinol. Stoffw. 2018 $\cdot 11: 73-80$ https://doi.org/10.1007/s41969-018-0043-6 Online publiziert: 21. September 2018 c Der/die Autor(en) 2018

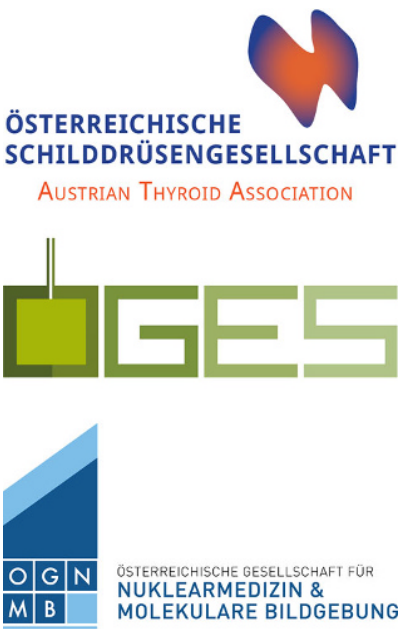

ACE der Österreichischen Gesellschaft für Chirurgie

CrossMark

\author{
Harald Dobnig ${ }^{1,12,14} \cdot$ Wolfgang Zechmann ${ }^{2,12} \cdot$ Michael Hermann $^{3,12,15}$. \\ Michael Lehner ${ }^{4,12}$. Dirk Heute ${ }^{5,12}$. Siroos Mirzaei ${ }^{6,13}$. Alois Gess ${ }^{7,14}$. \\ Vinzenz Stepan ${ }^{8,14}$. Günter Höfle ${ }^{9,14}$. Philipp Riss ${ }^{10,14,15}$. Andrea Simon ${ }^{11,15}$ \\ 'Schilddrüsen | Endokrinologie | Osteoporose Institut Dobnig, Graz, Österreich \\ ${ }^{2}$ Birgitz, Österreich \\ ${ }^{3}$ Chirurgische Abteilung, Krankenanstalt Rudolfstiftung, Wien, Österreich \\ ${ }^{4}$ Schilddrüsen- und Osteoporosepraxis Dr. Michael Lehner, Linz, Wels, Österreich \\ ${ }^{5}$ Private Krankenanstalt für Schilddrüsendiagnostik und Nuklearmedizin, Telfs, Österreich \\ ${ }^{6}$ Institut für Nuklearmedizin mit PET-Zentrum, Wilhelminenspital, Wien, Österreich \\ ${ }^{7}$ Klinische Abteilung für Endokrinologie und Stoffwechsel, Universitätsklinik für Innere Medizin III, Wien, \\ Österreich \\ ${ }^{8}$ Abteilung für Innere Medizin, Krankenhaus Elisabethinen, Graz, Österreich \\ ${ }^{9}$ Abteilung für Innere Medizin, Landeskrankenhaus Hohenems, Hohenems, Österreich \\ ${ }^{10} \mathrm{Klinische} \mathrm{Abteilung} \mathrm{für} \mathrm{Allgemeinchirurgie,} \mathrm{Universitätsklinik} \mathrm{für} \mathrm{Chirurgie,} \mathrm{Wien,} \mathrm{Österreich}$ \\ ${ }^{11}$ Klinische Abteilung für Allgemeinchirurgie, Universitätsklinik für Chirurgie, Graz, Österreich \\ ${ }^{12}$ Österreichische Schilddrüsengesellschaft (ÖSDG), Wien, Österreich \\ ${ }^{13}$ Österreichische Gesellschaft für Nuklearmedizin und Molekulare Bildgebung (OGNMB), Wien, Österreich \\ ${ }^{14}$ Österreichische Gesellschaft für Endokrinologie und Stoffwechsel (ÖGES), Innsbruck, Österreich \\ ${ }^{15}$ Arbeitsgemeinschaft für Chirurgische Endokrinologie (ACE) der Österreichischen Gesellschaft für \\ Chirurgie (OEGCH), Wien, Österreich
}

\title{
Radiofrequenzablation von Schilddrüsenknoten: Good Clinical Practice Empfehlungen
}

Die „Good Clinical Practice Empfehlungen“beziehen sich auf die Radiofrequenzablation (RFA) bezogene Ausbildung, Durchführung und Qualitätskontrolle sowie auf Standards hinsichtlich Vorund Nachsorge. Sie richten sich an Ärzte jeder Fachdisziplin, die die Technik der RFA zu erlernen beabsichtigen bzw. die Interventionen bereits durchführen, aber auch an Schilddrüsenspezialisten, die in die Vor- und Nachsorge von RFA-Patienten eingebunden sind. Die genannten Fachgesellschaften regen dringend an, diesen Empfehlungen nachzukommen.

RFA-Interventionisten, die sich an diesen Standards orientieren, werden auf einer mit obigen Fachgesellschaften verlinkten Homepage unter den „RFAZentren gemäß GCP-Empfehlungen der

Aus Gründen der besseren Lesbarkeit wurde im Text auf eine gendergerechte Schreibweise verzichtet. Sofern nicht anders vermerkt, gelten alle Bezeichnungen sowohl für Frauen als auch fürMänner.
ÖSDG/OGNMB/ÖGES/ACE der OEG$\mathrm{CH}^{\text {“ }}$ gelistet. Auf diese Weise soll eine Harmonisierung der RFA-Ausbildung und der Qualitätskontrolle in der Durchführung der Behandlung in Österreich gewährleistet sein.

\section{Einleitung}

\section{Thermoablative Techniken}

Die monopolare RFA zur Behandlung von Schilddrüsenknoten wurde 2002 von Prof. Dr. J.H. Baek, einem interventionell tätigen Radiologen am Asan Medical Center in Seoul, entwickelt. Mit Mai 2018 fanden sich mehr als 130 Einträge in der PubMed zum Thema „RFA und Schilddrüsenknoten“, sowohl in benigner als auch maligner Indikation. Von diesen Einträgen entfallen $u$. a. rund 40 auf Behandlungsstudien, 16 untersuchten die RFA im Vergleich oder in Kombination mit anderen Behandlungsoptionen,
29 sind Übersichtsartikel, 4 Metaanalysen, 28 Studien, die sich mit Grundlagen sowie Fragen zur Sicherheit und Komplikationen befassen.

Die Technik der monopolaren RFA wird in den Ländern, in denen sie angeboten wird, hauptsächlich im ambulanten Setting und von Spezialisten unterschiedlicher Fachdisziplinen durchgeführt. Sie bedarf einer speziellen Ausbildung und qualitativ guten Geräteausstattung. Die monopolare RFA wird meist mit einer wassergekühlten 18G-Sonde, die an einen Hochfrequenzgenerator angeschlossen ist, nach subkutaner und perikapsulärer Anästhesie mit Xylocain durchgeführt. Eine intravenöse Verabreichung von Analgetika, Sedativa oder anderen Medikamenten ist in aller Regel nicht notwendig, kommt aber in wenigen Zentren vor. Bei guter Technik ist ein nahezu schmerzloser Eingriff die Regel. Abgeleitet wird der Hochfrequenzstrom über geerdete „Pads“, die ventral auf 
beide Oberschenkel geklebt werden. Die Erdung ist seitens des Patienten mit keinen Missempfindungen verbunden. Während der RFA werden alle Patienten monitorisiert (EKG, Blutdruck, $\mathrm{pO}_{2}$ ).

Die Durchführung einer monopolaren RFA ist technisch anspruchsvoller, da der Knoten systematisch von kranial nach kaudal und von medial nach lateral in Freihandtechnik (Moving-shot-Technik) in einem vergleichsweise schmalen Ultraschallfenster unter steter Sicht der Sonde „abgearbeitet“ werden muss. Dies hat aber auch den Vorteil, dass man von der Knotengeometrie relativ unabhängig ist und den Knoten in seiner Gesamtheit gut behandeln kann. Erschwerend kommt hinzu, dass Knoten natürlich zu beiden Seiten der Trachea liegen können und so die sondenführende Hand einmal die linke, dann die rechte Hand ist und diese Bihändigkeit zuvor am Phantom eingeübt werden muss. Alternativ dazu kann der zur dominanten Hand ipsilateral gelegene Knoten allerdings auch von vorne transisthmisch bearbeitet werden, was aber auch einer Übung bedarf.

Nahezu das gesamte Wissen über die RFA als Behandlungsmethode bei Schilddrüsenknoten basiert auf den Erkenntnissen, die mit der „monopolaren“ Technik erzielt und publiziert wurden.

Ausgewählte Publikationen zum Thema monopolare RFA: [1-13].

Die bipolare RFA ist dagegen eine vergleichsweise junge RFA-Technik, mit $\mathrm{Pu}$ blikation der ersten Behandlungsstudien im Jahr 2016. Bei dieser Sonde ist der Plus- und Minuspol in die Spitze eingearbeitet, der Strom fließt daher lediglich durch das Knotengewebe und muss nicht abgeleitet werden. Der Hochfrequenzstrom zwischen diesen Polen baut ein relativ kugeliges Hitzefeld auf, innerhalb dessen das Gewebe überhitzt wird. Mit Abnahme des Wassergehalts des behandelten Knotengewebes steigt der Stromwiderstand, und die Geräteleistung wird nach unten reguliert.

Diese Technik ist manuell einfacher zu erlernen, da die Sondenspitze nur an einigen Stellen innerhalb des Knotens hier jedoch auch gezielt - platziert werden muss (Multiple-overlapping-shotTechnik). Die ersten Ergebnisse mit der bipolaren Technik sind sehr vielverspre- chend, jedoch fehlen zum gegenwärtigen Zeitpunkt noch Langzeitergebnisse und eine systematische Aufarbeitung größerer Patientenkollektive mit unterschiedlichen Knotencharakteristika.

Ausgewählte Publikationen zum Thema bipolare RFA: [14-16].

Andere thermoablative Verfahren haben im Vergleich zur RFA derzeit eine untergeordnete Bedeutung, da sie zugunsten unterlegener Ergebnisse gegenüber der RFA praktisch verlassen wurden (Laserablation) oder weil deren Wirksamkeit bzw. Komplikationsrate noch ungenügend belegt ist (Mikrowellenablation).

Die HIFU (,high intensity focused ultrasound") ist eine nichtinvasive Behandlungsmethode, bei der die thermische Koagulationsnekrose im Knotengewebe durch Bündelung von Ultraschallwellen bewerkstelligt wird. Die Komplikationsrate dürfte unter derjenigen anderer thermoablativer Verfahren liegen, jedoch können gemessen am Behandlungszeitrahmen auch nur vergleichsweise kleine Knotenvolumina behandelt werden. Dorsal gelegene Knoten sind topographisch ungünstig. Ein derzeit noch unzureichend gelöstes Problem stellen die Schmerzen während der Behandlung dar, die durch Kapselreizung, aber auch durch Irritation des Plexus brachialis auftreten und nur schwer zu vermeiden sind. Aus diesem Grund wird in manchen Zentren die HIFU unter Vollnarkose/Analgosedierung durchgeführt.

Ausgewählte Publikationen zur Übersicht thermoablativer Techniken: [17, 18].

\section{Offene Fragen}

Alle thermoablativen Behandlungsverfahren haben hinsichtlich des Auftretens von Knotenrezidiven die fehlende (bipolare RFA, Mikrowellenablation, HIFU) oder spärlich vorliegende (Laserablation, monopolare RFA) Langzeitdokumentation gemeinsam. Es steht außer Zweifel, dass bei den meisten Patienten die Knotenverkleinerung eine zuvor vorhandene lokale Symptomatik über lange Zeit verbessert und während dieser Zeit die Schilddrüsenfunktion in aller Regel er- halten bleibt. $\mathrm{Zu}$ welchem Prozentsatz unzureichend behandelte Knotenanteile langfristig wachsen und wieder eine Symptomatik verursachen oder vielleicht neue Knoten in der gleichen Schilddrüse einer Intervention bedürfen, ist noch unbekannt. Auch wenn in solchen Fällen ein (vermutlich kleinerer) Zweiteingriff möglich sein dürfte, wird dies die KostenNutzen-Rechnung gegenüber Operation oder Radiojodtherapie beeinflussen.

Die Frage, inwieweit thermoablatierte Schilddrüsenknoten ein mögliches Problem im Falle einer doch notwendigen nachfolgenden Operation darstellen, ist noch unbeantwortet. Es ist jedoch vorhersehbar, dass Verwachsungen dort auftreten können, wo die ursprüngliche „Hitzewelle“ die Schilddrüsenkapsel und eventuell die Nachbarschaft in einem signifikanten Ausmaß erreicht hat.

Alleine schon aufgrund der langjährigen Erfahrungen, die mit der Schilddrüsenchirurgie vorliegen, wird sich jede thermoablative Methode hinsichtlich Effektivität, Komplikationen, Patientenzufriedenheit und Kosten-Nutzen-Rechnung am aktuellen Goldstandard messen müssen.

\section{Ausbildung}

Voraussetzung für eine standardisierte RFA-Schulung (- Abb. 1)

- 40 dokumentierte "transisthmische " Interventionen (beinhaltet Punktionen, Stanzbiopsien, Alkoholablationen), davon idealerweise 5 Alkoholablationen.

Anmerkung: Diese Vorbereitungsphase soll sicherstellen, dass 1.) die Position des Arztes zum Hals bzw. Knoten (kopfseitig mit dem Monitor direkt vor Augen) und 2.) Eingriffe von transisthmisch kommend beides unabdingbare Voraussetzungen für die Durchführung der RFA - ausreichend praktiziert und erfahren werden. Außerdem ist das Erlernen einer gewissen Bihändigkeit von großem Vorteil, da dann mit der kopfseitigen Position des Arztes links- und rechtsseitig gelegene Kno- 


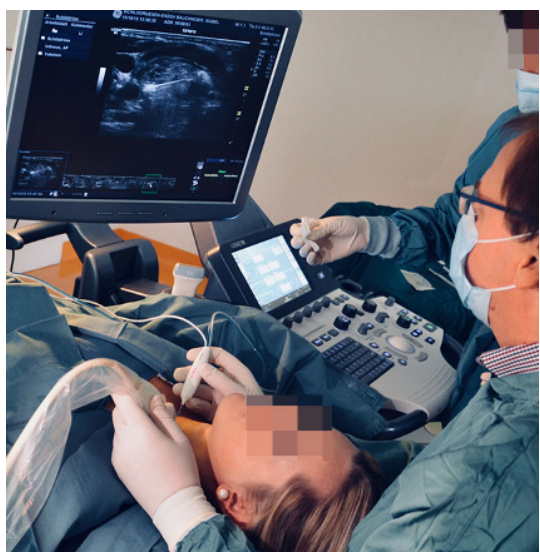

Abb. 1 A Typisches Setting bei einer Radiofrequenzablation eines Schilddrüsenknotens. Der Arzt sitzt an der Kopfseite des Patienten, den Monitor direkt vor Augen, arbeitet idealerweise in dieser Position Knoten zu beiden Seiten ab. Eine Assistenz monitorisiert den Patienten und bedient den Hochfrequenzgenerator, die zweite assistiert direkt dem Arzt. Ein typischer Eingriff dauert 30 min, gefolgt von einer Nachbeobachtungszeit

ten gleichermaßen behandelt werden können.

- Erwünschte Hospitation in einem endokrin-chirurgischen Zentrum (z.B. 2 Operationsvormittage) zur Erfassung der anatomischen Strukturen (insbesondere Nerven- und Gefäßverlauf) im Halsbereich.

\section{Standardisierte RFA-Einschulung}

- Anwesenheit bei 8-10 RFAs bei einem erfahrenen Kollegen (der mindestens 100 RFAs durchgeführt haben muss)

- Begleitende, ausführliche theoretische Schulung (inklusive Demonstration von Langzeitverläufen, Besprechung möglicher Komplikationen und anatomischer Besonderheiten, Sichtung von Videomaterial)

- Praktische Schulung (mit Übungen am Phantom)

- Einschulung des Assistenzpersonals an zumindest einem Interventionstag

- 4 betreute RFAs vor Ort im Beisein des ausbildenden Kollegen Anmerkung: Eine solche standardisierte RFA-Schulung ermöglicht eine maximale individuelle und intensive Vorbereitung, die so im Rahmen eines größeren „Kurses“ nie gegeben wäre. In solchen Kursen könnten letztlich keine praktischen Inhalte vermittelt werden, die in diesem Fall besonders wichtig sind. Deshalb sollte idealerweise eine 1:1- bis maximal 1:2-Schulung erfolgen.

Kurse werden prinzipiell in Südkorea und Italien (monopolare RFA) bzw.

in Deutschland (bipolare RFA) und in Österreich (monopolare und bipolare RFA) angeboten. Nach derzeitigem Kenntnisstand sind die Kurse in Südkorea und Österreich die zeitlich aufwendigsten und erstrecken sich über insgesamt 3 Tage.

Im Österreichischen Schulungsmodell wird im Anschluss an die Ausbildung das Coaching „vor Ort“ angeboten und wird generell nachfolgend eine Teilnahme am Qualitätsprogramm der AG-RFA erwartet.

\section{Zertifikat}

Als Beleg über die Vorbereitungs- bzw. Ausbildungsphase wird seitens der AGRFA im Namen der ÖSDG/OGNMB/ ÖGES/ACE der OEGCH ein entsprechendes Zertifikat ausgestellt.

\section{Laufende Qualitätskontrolle}

- Verwendung der einheitlichen „Patienteninformation und -einverständniserklärung", um einen gleichlautenden Standard zu gewährleisten (wird den Teilnehmern von der AG-RFA zur Verfügung gestellt).

- Fallfrequenz: Wie allgemein bei operativen Verfahren erhöht auch eine regelmäßig durchgeführte RFA die Sicherheit und Effektivität des Eingriffes. Die oben involvierten Fachgesellschaften gehen davon aus, dass man bei mindestens 40 therapierten Fällen pro Jahr von einer „ausreichenden“ Frequenz sprechen kann, die eine gute Behandlungsqualität sicherstellen sollte.

- Führung einer Falldokumentation aller RFA-Interventionen nach einheitlichen Kriterien (zur Falldokumentation gibt es ein entsprechendes Excel-File über die AG-RFA)
- Führung einer Videodokumentation aller RFA-Interventionen inklusive Aufnahme einer Sequenz ca. 5 min nach Abschluss der RFA-Behandlung zur Dokumentation des Endstatus.

- Anbindung eines RFA-Zentrums an ein endokrin-chirurgisches Zentrum, um im Falle einer auftretenden Komplikation einen rasch zur Verfügung stehenden Ansprechpartner zu haben. Der Name der kooperierenden Institution sowie der involvierten Kollegen muss der AG-RFA bekannt gemacht werden und wird ebenfalls auf der Homepage veröffentlicht.

- Mit Abschluss des Kalenderjahres Übermittlung eines standardisierten jährlichen schriftlichen Kurzberichts, aus dem die Ergebnisse und die Komplikationen hervorgehen (das Formular wird von der AG-RFA zur Verfügung gestellt).

- Die RFA-Zentren, die sich freiwillig den qualitätssichernden Maßnahmen unterziehen, sind mit der Überprüfung der übermittelten Datenqualität in Form angekündigter Audits (alle 2-3 Jahre), die von der AG-RFA organisiert werden, einverstanden. Um die Organisation dieser (Non-profit-) Untersuchungen zu ermöglichen, werden die teilnehmenden Zentren gebeten (gegen Rechnungslegung), jährlich einen Beitrag von $€ 500$,- auf das Konto der AG-RFA der ÖSDG zu überweisen. Damit sollen auch andere Kosten für Homepageerstellung, Dateneingabe etc. abgedeckt werden.

\section{Bestätigung über die laufende Qualitätskontrolle}

Über die teilnehmenden Zentren, die jährlich eingegangenen Berichte zur Qualitätssicherung sowie die Ergebnisse der Audits wird es auf einer eigenen Homepage, die idealerweise mit allen teilnehmenden Fachgesellschaften verlinkt ist, entsprechende Informationen geben. 

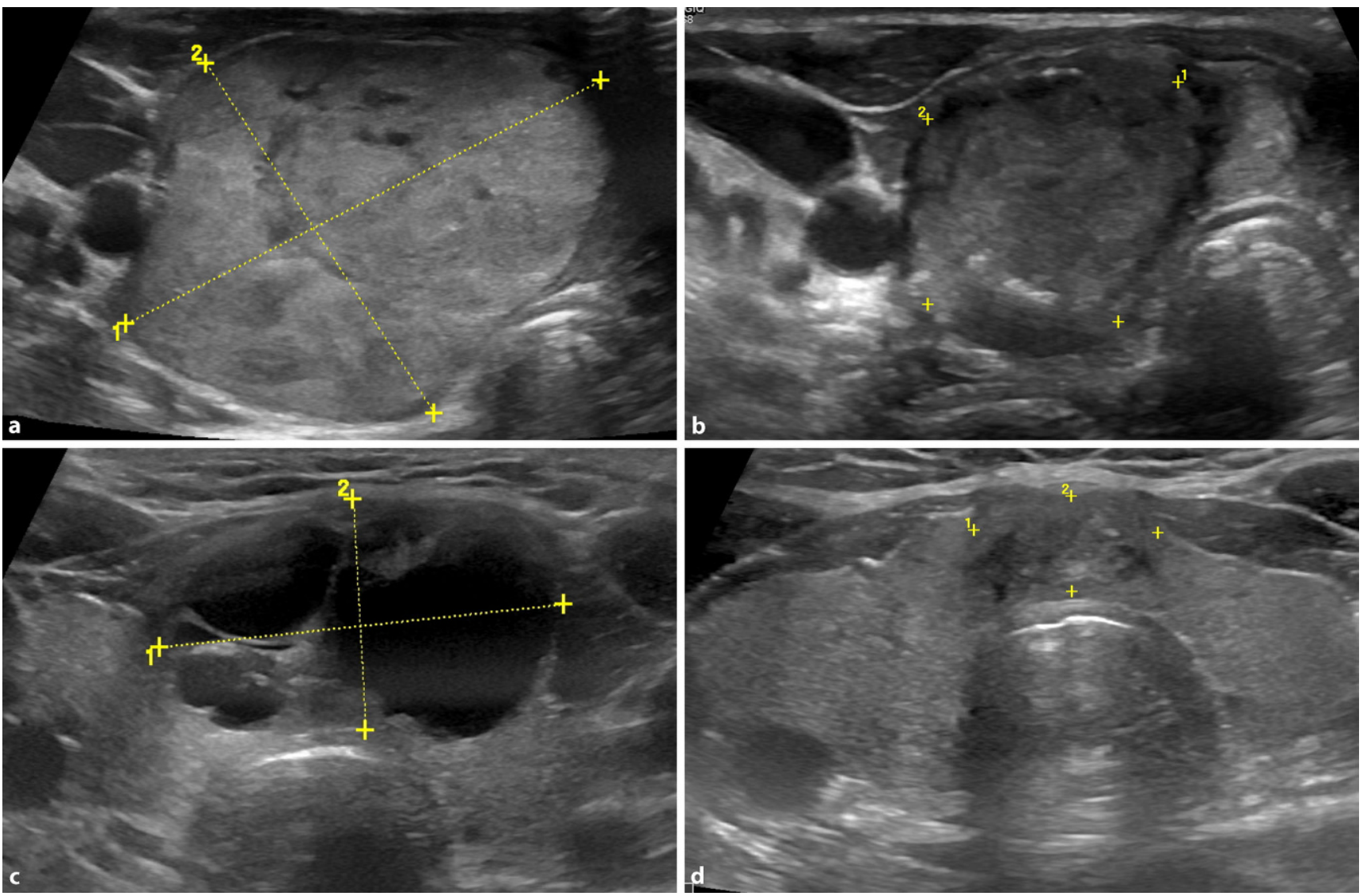

Abb. $2 \Delta$ a und b Beispiel einer Radiofrequenzablation (RFA) eines soliden Knotens vor und 12 Mon. nach der Behandlung. a 50-jährige Patientin, 5-facherVolumenzuwachs des Knotens in 8 Jahren, mehrfache Operationsempfehlung, visuell der Knoten deutlich sichtbar, Symptomatikscore 4 von 10; Knotenvolumen: $40 \mathrm{ml}$. b Nach 12 Monaten der Knoten nur noch palpabel, Symptomatikscore 0, Knotenvolumen 8,2 $\mathrm{ml}$ (-80\%). Dieses Beispiel zeigt auch, dass das exakte Setzen der Messmarken, die sich an der initial längsten Querachse orientieren, wichtig ist für die Erfassung des Verlaufs des Knotenvolumens. c und d Beispiel einer RFA eines zystischen Kolloidknotens. c Prätracheales, kompensiertes zystisches autonomes Adenom, hochvisköser Inhalt, Knotenvolumen 10,6 ml, TSH 0,6 UU/ml, Symptomatikscore 4 (von 10), visueller Knotenscore 3 (von 3), Operation empfohlen. $\mathbf{d}$ Nach 12 Monaten visueller und Symptomatikscore 0, transformiertes Knotenrestgewebe 1,2 ml (-89\%), TSH 1,0 $\mathrm{uU} / \mathrm{ml}$

\section{Indikation}

\section{Publizierte Indikationen}

Folgende prinzipiellen Indikationen finden sich in den Guidelines bzw. Stellungnahmen der angeführten Gesellschaften:

- Korean Society of Thyroid Radiology (KSThR), 2009 und 2011 Guideline [19]

- Italian Expert Opinion Statement 2015 [20]

- American Association of Clinical Endocrinologists (ACCE) 2016 Guideline

- American College of Endocrinology (ACE) 2016 Guideline

- Associazione Medici Endocrinology (AME) 2016 Guideline [21]
- National Institute for Health and Care Excellence (NICE) 2016 Guideline (https://www.nice.org.uk/guidance/ ipg562)

- Österreichische Schilddrüsengesellschaft 2016 Offizielle Stellungnahme (https://www.kup.at/kup/pdf/13399. pdf)

- Benigner Knoten mit belastender Symptomatik u/od. optisch störend - Benigner Knoten mit kontinuierlichem Wachstum (gleichzeitig $>2 \mathrm{~cm}$ Durchmesser) und begleitender Symptomatik

- Autonomer Knoten, wenn eine Radiojodtherapie (RJT) oder Operation kontraindiziert oder nicht gewünscht ist
- Differenziertes Schilddrüsenkarzinom: bei lokalem Rezidiv und hohem Operationsrisiko bei gleichzeitiger Jodrefraktärität (palliativer Therapieansatz)

Im Diskussionsstadium: „low-risk“ papilläres Mikrokarzinom - in Fällen, wo aktuell eine "active surveillance“ diskutiert wird (Topographie innerhalb der Schilddrüse, cN0, kein Hinweis auf Multifokalität oder Invasivität, Kontraindikation [KI] für OP) [22]. 


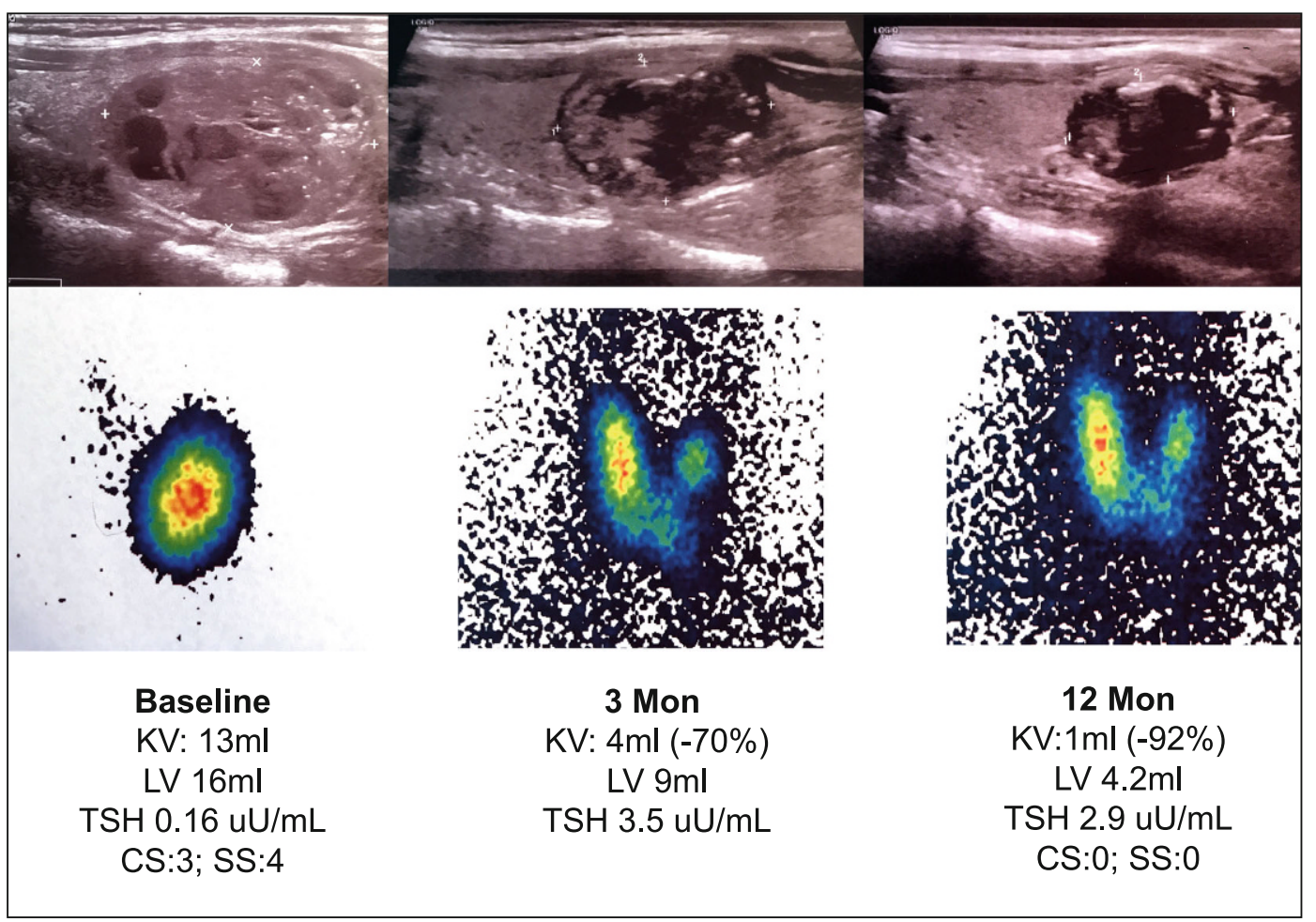

Abb. $3 \Delta$ Beispiel einer Radiofrequenzablation (RFA) eines autonomen Adenoms. 48-jährige Patientin, autonomes Adenom seit Jahren bekannt, zuletzt deutliches Wachstum und Entwicklung einer latenten Hyperthyreose. Operation wurde empfohlen. Der Ausgangsbefund ist ein $13 \mathrm{ml}$ großes, $40 \%$ zystisches, autonomes Adenom, welches den Großteil des linken Schilddrüsenlappens einnimmt, funktionell dekompensiert. Die Ultraschallbilder zeigen den Sagittalschnitt durch den linken Schilddrüsenlappen mit den morphologischen Veränderungen 3 und 12 Monate nach der RFA. Da das behandelte autonome Adenom nach der RFA bindegewebig umgebaut wird, wird aus dem in der Technetium-99m-Szintigraphie vormals ",heißen“ Knoten ein „warmer" oder idealerweise sogar ein „kalter" Knoten. KV Knotenvolumen, LV Lappenvolumen, CS visueller Knotenscore (bis Grad 3); SS Symptomatikscore (bis Grad 10)

\section{Praktische Erfahrungen zur Indikation und Einschränkung}

\section{Monopolare RFA}

Allgemein: Behandlung von 1-3 Knotenbildungen, auch bilateraler Eingriff in einer Sitzung möglich (jedoch abhängig von beidseitiger Knotentopographie).

\section{Gute Indikationen}

- Zystische, oder prädominant zystische Knoten (inkl. „Kolloidzysten“;

- Abb. 2) sind die beste Indikation für eine RFA! (Volumen auch $>30 \mathrm{ml}$ möglich).

- falls eine Punktion oder Alkoholablation nicht zum gewünschten Erfolg führt, oder nicht sinnvoll/möglich ist.

Anmerkung: „Akute Blutungszysten" sollten jedenfalls immer erst vollständig und ultraschallgezielt punktiert werden, bevor eventuell weitere Maßnahmen getroffen werden. Sogenannte „benigne“
Zysten, die ausschließlich glatt begrenzt sind, sprechen meist gut auf eine Alkoholverödung an. Mit einer Alkoholablation kann jedoch immer nur der zystische, nicht aber der solide Knotenanteil (dies ist obsolet!) behandelt werden. Nach Alkoholablation kommen in ca. $30 \%$ Spätrezidive vor.

- Solider und gemischter Knoten bis ca. $30 \mathrm{ml}$ (als Einmalbehandlung)

- Autonome Adenome - solide oder zystisch bis ca. 12 bis $15 \mathrm{ml}$ (als Einmalbehandlung; • Abb. 3)

- Wachsende Knoten - wenn sie eine beginnende Symptomatik verursachen (immer nach Wiederholung einer Biopsie mit Feinnadelpunktion (FNP) oder Feinnadelkapillarzytologie (FNCC), oder ggf. Stanzbiopsie!)

- Z.n. Schilddrüsenoperation

- Erhöhtes Narkoserisiko

- Bekannte Keloidneigung!
Eingeschränkte oder keine Indikationen

- Zytologisch Bethesda >II oder andere Form des Malignitätsverdachts

- Weit kaudal reichender Knoten, nicht in toto zu erreichen

- Eher schmaler und zapfenförmig tief nach dorsal reichender Knoten

- Prominente ventrale Gefäße in der Behandlungsebene

- Große solide und gemischte Knoten $>30 \mathrm{ml}$ (als Einzeleingriff)

- Diffuse Schilddrüsen-Vergrößerung mit mehrfachen Knotenbildungen (wenn auch eine erfolgreiche RFA kein zufriedenstellendes Endergebnis erwarten lässt)

- Autonomes Adenom >15 ml (als Einzeleingriff). Hier jedoch oft gute Ergebnisse mit nachfolgender lowdose $\mathrm{J}^{131}$-Therapie möglich.

- Multifokale Autonomie (individuell einzuschätzen: von Anzahl, Größe der Knoten, Patientenalter abhängig) 
- Hashimoto-Thyreoiditis

- Morbus Basedow

- Schrittmacherträger (gilt für monopolare RFA)

- Schwangerschaft (gilt für monopolare RFA)

\section{Bipolare RFA}

\section{Gute Indikationen}

- Großer, solider Knoten - speziell bei topographisch schwierigeren Lagen manchmal leichter zu behandeln als mit monopolarer RFA

- Palliative Volumenreduktion (nur in selektiven Fällen)

- Schwangerschaft: keine KI wie bei monopolarer RFA

- Schrittmacherträger: keine KI wie bei monopolarer RFA

\section{Eingeschränkte Indikationen}

- Knoten mit höherem Einblutungsrisiko (größerer Sondendurchmesser)

- Knoten unterschiedlicher Größe, wenn gleichzeitige Behandlung von mehr als einem Knoten geplant ist und mit einer Sondengröße alleine nicht zufriedenstellend behandelt werden kann (höhere Materialkosten)

- Kleineres autonomes Adenom (Energiedichte mit monopolarer Sonde höher)

\section{Patientenaufklärung}

Die eingehende, durchgeführte Aufklärung über die zur Verfügung stehenden Behandlungsmöglichkeiten sollte prinzipiell immer und unabhängig vom Spezialgebiet des aufklärenden Facharztes aus dem Arztbrief hervorgehen.

Die Operation von benignen symptomatischen oder störenden Schilddrüsenknoten stellt die „Standardtherapie“ in der Behandlung dar. Alternativ dazu kann bei geeigneten Patienten, die eine Operation nicht wünschen oder bei denen eine solche mit einem erhöhten Risiko verbunden ist, nach eingehender Aufklärung und Darlegung aller in Frage kommenden Behandlungsverfahren (Operation, Radiojodtherapie, Alkoholablation bei zystischen Knoten, Radiofrequenzablation), inklusive deren Vor- und Nachteile, eine RFA angeboten werden. Idealerweise finden sich im
Arztbrief Hinweise für eine Begründung des Therapiewunsches sowie eine kurze Erläuterung des Therapieziels.

Sollte sich der Patient für eine RFA entscheiden, muss zusätzlich, wie auch im Rahmen einer Operation, eine unterschriebene, einheitlich formulierte Patienteninformation und -einverständniserklärung vorliegen (wird den Teilnehmern von der AG-RFA zur Verfügung gestellt).

\section{Vorsorge}

Die erste Selektion für ein geeignetes Behandlungsverfahren obliegt naturgemä $\beta$ dem betreuenden Schilddrüsenspezialisten, der den Patientenfall in seiner Gesamtheit kennt und diesen inklusive der Therapieoptionen am besten einschätzen kann.

Für den nächsten Schritt in der Patientenauswahl kann sich aus praktischen Gründen eine Vorab-Evaluierung durch den RFA-Interventionisten als sehr nützlich erweisen. Oft kann nämlich bereits nach Durchsicht der Sonographiebilder und der wesentlichsten Befunde eine RFA vorab ausgeschlossen werden - oder aber eine Aussage getroffen werden, ob als nächster Schritt eine persönliche Vorstellung sinnvoll erscheint. Erfahrungsgemäß muss besonders häufig dort eine entsprechende Absage getätigt werden, wo sich der Patient aus eigenem Antrieb und oft begleitet von falschen Vorstellungen über erreichbare Therapieziele meldet.

Idealerweise tritt daher der Schilddrüsenspezialist mit dem RFA-Interventionisten in Kontakt (z. B. Übermittlung eines aussagekräftigen Befundes inklusive Labor, idealerweise mit Szintigraphiebefund und Sonographiebildern).

\section{Zytologischer Befund}

Es ist wichtig darauf hinzuweisen, dass die RFA-Behandlung nur eine vorgeschaltete zytologische, nicht aber eine histologische Abklärung eines Knotens erlaubt. Deshalb ist bei „kalten“ oder „warmen" Knotenbildungen eine aussagekräftige ultraschallgezielte Punktion mit einem benignen zytologischen Ergebnis vor der RFA notwendig, damit die geforderte Sicherheit des zytologischen Befundes gewährleistet ist.

Man geht heute davon aus, dass eine gut durchgeführte, aussagekräftige ultraschallgezielte Feinnadelpunktion (FNP) eine Wahrscheinlichkeit von 1-2\% für einen „falsch-negativen“ zytologischen Befund aufweist (siehe ATA-Guidelines). In einer Untersuchung des AKH Wien (Mitteilung Prof. Dr. Riss) lag dieser Prozentsatz bei 5,9\% und wird vor allem auf das Vorliegen multinodöser Strumen im untersuchten Patientenkollektiv zurückgeführt.

$\mathrm{Da}$ es alleine aus verschiedenen methodologischen Gründen nie einen 100\%igen Ausschluss eines Karzinoms geben kann, wird folgendes Vorgehen vorgeschlagen:

Es sollen jedenfalls nur solche Knoten mit einer RFA behandelt werden, für die eine negative Zytologie und ein unauffälliger Ultraschallstatus der zervikalen Lymphknoten vorliegen. Bei Knoten, die seit einer Punktion gewachsen sind, oder solchen, die sonographische Auffälligkeiten aufweisen (z. B. Mikrokalk, „taller than wide“, unregelmäßige Begrenzung), sollte eine zweite, ultraschallgezielte Punktion durchgeführt werden. Eine bestätigte benigne Zytologie schließt laut ATA-Guideline eine Malignität praktisch aus.

Eine Fotodokumentation der Nadelspitze zum Zeitpunkt der Punktion ist gefordert. Bei „heißen“ Knotenbildungen ist, bei sonst fehlenden Hinweisen auf Malignität, eine Feinnadelpunktion vor der RFA nicht notwendig.

Es ist also von großer Wichtigkeit, dass zusätzlich zum zytologischen Befund immer auch der sonographische Gesamtaspekt des Knotens inklusive dem obligatorischen Ultraschallstatus der Halslymphknoten in die Evaluierung eingeht.

\section{Laryngoskopische Evaluierung des Stimmbandstatus}

Für eine routinemäßige Empfehlung der Erhebung eines Stimmbandstatus vor und nach der RFA gibt es bislang keine fundierte wissenschaftliche Literatur. In einer Metanalyse wird von einem extrem niedrigen Risiko permanenter Stimmbandlähmung berichtet (ca. 0,05\%), ein 
1. Sichtung Vorbefunde

2. Labor (TSH, fT4, fT3, TPOAK, TGAK, Tg, fakultativ Calcitonin, PTH, Kalzium und Gesamteiweiß, Gerinnung)

3. Gutartigkeit durch ultraschallgezielte FNP oder FNCC erwiesen? Passt die Knotenmorphologie dazu?

4. Genauer Ultraschallbefund

- Lymphknotenstatus?

- Prominente Gefäße in Ebene der Sondenführung?

- Eintrittspforte für lokale Anästhesie?

- Knoten in toto erreichbar?

- Anzahl und Lage der Eintrittspforten?

- Lagebeziehung des Knotens zu Nervus vagus und Trachea?

- Lagebeziehung des Knotens zum „danger triangle“?

- Knoten- und Lappenvolumina mit Dokumentation der Messmarken in Horizontal- und Sagittalebene

- Bei zystischen Knoten: Aspiration vor der RFA erforderlich? Wahrscheinlichkeit für Kolloidzyste? Einblutungsrisiko?

5. Auswirkungen der RFA auf Spontanfunktion bzw. Medikation nach dem Eingriff? (ausreichendes Restgewebe vorhanden? Wahrscheinlichkeit einer low-dose RJT als Nachbehandlung bei autonomen Adenom?)

6. Symptomatik-Score (VAS 1-10) Frage an den Patienten: „In welchem Ausmaß beeinträchtigt sie der Knoten, wobei sich diese Beeinträchtigung auf funktionelle, visuelle, oder psychische Aspekte (oder eine Kombination derselben) bezieht?"

7. Visueller Knotenscore $(0=$ Knoten nicht palpabel, $1=$ Knoten palpabel, $2=$ Knoten bei Reklination des Kopfes oder beim Schlucken sichtbar

$3=$ Knoten mit freiem Auge gut sichtbar)

8. Wichtige (relative oder absolute) Kontraindikationen vorhanden?

- Blutungsneigung, Antikoagulation?

- Schrittmacherträger?

- Schwangerschaft? Stillt die Patientin?

- Allergien gegenüber lokalen Anästhetika?

- Schwerwiegende HWS-Probleme? (wegen der erforderlichen Überstreckung)

9. Patientenaufklärung im Arztbrief dokumentiert?

10. Patienteninformation und -einverständniserklärung ausgehändigt?

11. HNO-Befund mit Stimmbandstatus eingefordert?

FNP Feinnadelpunktion, FNCC Feinnadelkapillarzytologie, PTH Parathormon, RFA Radiofrequenzablation, VAS visual analogue scale (visuelle Analogskala)

„Publication-Bias“ kann aber bei diesem vulnerablen Thema aufgrund fehlender systematischer Untersuchungen nicht ausgeschlossen werden [23].

Daher: Da eine Recurrensparese funktionell gut kompensiert sein kann und sprachlich nicht auffällig sein muss, wird - in Analogie zur Schilddrüsenoperation - eine Erhebung des laryngoskopischen Stimmbandstatus bei allen Patienten vor der RFA und möglichst zeitnah nach der RFA gefordert. Die Patienten werden gebeten, den zweiten Befund spätestens zur Dreimonatskontrolle vorzulegen. Auf diese Weise soll eine wichtige bisherige Lücke in der Gesamtbeurteilung der Behandlungsqualität geschlossen werden.

\section{RFA-Checkliste für den Interventionisten}

Siehe • Tab. 1.

\section{Nachsorge}

Im Rahmen einer strukturierten Nachsorge sollten Kontrollen mit Ultraschall 3 und 12 Monate nach der RFA erfolgen, um die unmittelbaren Auswirkungen der RFA-Behandlung zu dokumentieren. Die Patienten werden gebeten, zur Dreimonatskontrolle den postinterventionellen HNO-fachärztlichen Befund des Stimmbandstatus mitzubringen. Sind die Schilddrüsenkontrollen (und weitere empfohlene im 1- bis 2-jährigen Abstand) seitens des Patienten beim RFA-durchführenden Arzt hingegen nicht möglich, sollte der nachsorgende Schilddrüsenspe- zialist in seinem Befund idealerweise auf folgende Punkte eingehen:

- Stimmbandstatus nach der RFAIntervention

- Volumetrie des/der behandelten Knoten(s) und Volumetrie beider Schilddrüsenlappen.

Idealerweise gibt der RFA-durchfüh rende Arzt dem Patienten relevante Ultraschallbilder mit. Daraus ersieht der Schilddrüsenspezialist die Positionierung der Messmarken, die für die Volumetrie der Schilddrüsenlappen und des Knotens verwendet wurden, und kann so eine valide Verlaufsmessung durchführen. Cave! Der bindegewebig transformierte Knoten ist (bedingt durch den Verlust seiner vesikulären Struktur und damit Reduktion schallreflektierender Grenzflächen) deutlich echoarm (!) und häufig avaskulär 
sowie auch deutlich kleiner als in einem Vorbefund beschrieben. Er kann auch Mikrokalk beinhalten. Manche bindegewebig geschrumpfte Knoten können auch vaskularisiert sein. Der Knoten hat zudem entweder eine "zytologisch benigne Vergangenheit", oder war funktionell im Szintigramm „autonom“ (und damit benigne). Es ist also essenziell, dass sich der Schilddrüsenspezialist in diese morphologischen Besonderheiten nach einer RFA-Behandlung ,einsieht“ und sie richtig interpretiert, da der Patient sonst unnötig beunruhigt werden könnte.

- Zusätzlich sollten TSH, fT4 und fT3 wie auch zumindest zur Dreimonatskontrolle die TPOAK und TGAK bestimmt werden. Wurde mit der RFA ein toxischer Knoten behandelt, empfiehlt sich zur Dreimonatskontrolle die zusätzliche Durchführung einer SD-Szintigraphie.

- Ganz generell wird auch im Anschluss an die beiden Kontrollen nach der RFA eine 1- bis 2-jährige Ultraschalluntersuchung der Schilddrüse und des zervikalen Lymphknotenstatus empfohlen.

\section{Korrespondenzadresse}

\section{Harald Dobnig}

Schilddrüsen | Endokrinologie | Osteoporose Institut Dobnig

Jakob-Redtenbachergasse 10, $8010 \mathrm{Graz,}$

Österreich

harald.dobnig@hormoninstitut-dobnig.at

\section{Alois GessI}

Klinische Abteilung für Endokrinologie und Stoffwechsel, Universitätsklinik für Innere Medizin III

Währinger Gürtel 18-20, 1090 Wien, Österreich alois.gessl@meduniwien.ac.at

Funding. Open access funding provided by Medical University of Vienna.

\section{Einhaltung ethischer Richtlinien}

Interessenkonflikt. H. Dobnig, W.Zechmann, M. Hermann, M. Lehner, D. Heute, S. Mirzaei, A. Gessl, V. Stepan, G. Höfle, P. Riss und A. Simon geben an, dass kein Interessenkonflikt besteht.
Dieser Beitrag beinhaltet keine von den Autoren durchgeführten Studien an Menschen oder Tieren.

Open Access. Dieser Artikel wird unter der Creative Commons Namensnennung 4.0 International Lizenz (http://creativecommons.org/licenses/by/4.0/deed. de) veröffentlicht, welche die Nutzung, Vervielfältigung, Bearbeitung, Verbreitung und Wiedergabe in jeglichem Medium und Format erlaubt, sofern Sie den/die ursprünglichen Autor(en) und die Quelle ordnungsgemäßnennen, einen Link zur Creative Commons Lizenz beifügen und angeben, ob Änderungen vorgenommen wurden.

\section{Literatur}

1. Kim YS, Rhim H, Tae K, Park DW, Kim ST (2006) Radiofrequency ablation of benign cold thyroid nodules: initial clinical experience. Thyroid 16(4):361-367

2. Jeong WK, BaekJH, Rhim H, Kim YS, KwakMS, Jeong $\mathrm{HJ}$ et al (2008) Radiofrequency ablation of benign thyroid nodules: safety and imaging follow-up in 236 patients. Eur Radiol 18(6):1244-1250

3. Baek JH, Kim YS, Lee D, Huh JY, Lee JH (2010) Benign predominantly solid thyroid nodules: prospective study of efficacy of sonographically guided radiofrequency ablation versus control condition. Ajr Am J Roentgenol 194(4):1137-1142

4. Sung JY, Baek JH, Kim KS, Lee D, Yoo H, Kim JK et al (2013) Single-session treatment of benign cystic thyroid nodules with ethanol versus radiofrequency ablation: a prospective randomized study. Radiology 269(1):293-300

5. Dobrinja C, Bernardi S, Fabris B, Eramo R, Makovac P, Bazzocchi Getal (2015) Surgical and pathological changes after radiofrequency ablation of thyroid nodules. Int J Endocrinol. https://doi.org/10.1155/ 2015/576576

6. Che Y, Jin S, Shi C, Wang L, Zhang X, Li Y et al (2015) Treatment of benign thyroid nodules: comparison of surgery with radiofrequency ablation. Ajnr Am J Neuroradiol 36(7):1321-1325

7. Deandrea M, Sung JY, Limone P, Mormile A, Garino F, Ragazzoni F et al (2015) Efficacy and safety of radiofrequency ablation versus observation for nonfunctioning benign thyroid nodules: a randomized controlled international collaborative trial. Thyroid 25(8):890-896

8. Valcavi R, Tsamatropoulos P (2015) Health-related quality of life after percutaneous radiofrequency ablation of cold, solid, benign thyroid nodules: a 2-year follow-up study in 40 patients. Endocr Pract 21(8):887-896

9. Cesareo R, Pasqualini V, Simeoni C, Sacchi M, Saralli E, Campagna G et al (2015) Prospective study of effectiveness of ultrasound-guided radiofrequency ablation versus control group in patients affected by benign thyroid nodules. J Clin Endocrinol Metab 100(2):460-466

10. Dobnig H, Amrein K (2018) Monopolar Radiofrequency ablation of thyroid nodules: a prospective Austrian single-center study. Thyroid 28(4):472-480

11. Chen F, Tian G, Kong D, Zhong L, Jiang T (2016) Radiofrequency ablation for treatment of benign thyroid nodules: A PRISMA-compliant systematic review and meta-analysis of outcomes. Medicine (Baltimore) 95(34): :4659

12. Jung SL, Baek JH, Lee JH, Shong YK, Sung JY, Kim KS et al (2018) Efficacy and safety of radiofrequency ablation for benign thyroid nodules: a prospective multicenter study. Korean J Radiol 19(1):167-174
13. Ha EJ, Baek JH, Kim KW, Pyo J, Lee JH, Baek SH et al (2015) Comparative efficacy of radiofrequency and laser ablation for the treatment of benign thyroid nodules: systematic review including traditional pooling and bayesian networkmeta-analysis.JClin Endocrinol Metab 100(5):1903-1911

14. LiXL, Xu HX, LuF, YueWW, Sun LP, BoXWetal (2016) Treatment efficacy and safety of ultrasoundguided percutaneous bipolar radiofrequency ablation for benign thyroid nodules. Br J Radiol 89(1059):20150858. https://doi.org/10.1259/bjr 20150858

15. Kohlhase KD, Korkusuz Y, Groner D, Erbelding C, Happel C, Luboldt W et al (2016) Bipolar radiofrequency ablation of benign thyroid nodules using a multiple overlapping shot technique in a 3-month follow-up. Int J Hyperthermia 32(5):511-516

16. Korkusuz Y, Mader A, Groner D, Ahmad S, Mader OM, Grunwald F et al (2017) Comparison of monoand bipolar radiofrequency ablation in benign thyroid disease. World J Surg 41(10):2530-2537

17. Barile A, Quarchioni S, Bruno F, lerardi AM, Arrigoni $\mathrm{F}$, Giordano AV et al (2018) Interventional radiology of the thyroid gland: critical review and state of the art. Gland Surg 7(2):132-146. https://doi.org/10. 21037/gs.2017.11.17

18. Gharib H, Hegedus L, Pacella CM, Baek JH, Papini E (2013) Clinical review: nonsurgical, image-guided, minimally invasive therapy for thyroid nodules. JClin Endocrinol Metab 98(10):3949-3957

19. Na DG, Lee JH, Jung SL, Kim JH, Sung JY, Shin JH et al (2012) Radiofrequency ablation of benign thyroid nodules and recurrent thyroid cancers: consensus statement and recommendations. Korean J Radiol 13(2):117-125

20. Garberoglio R, Aliberti C, Appetecchia M, Attard M, Boccuzzi G, Boraso F et al (2015) Radiofrequency ablation for thyroid nodules: which indications? The first Italian opinion statement. J Ultrasound 18(4):423-430

21. Gharib H, Papini E, Garber JR, Duick DS, Harrell RM, Hegedus L et al (2016) American association of clinical endocrinologists, American college of endocrinology, and Associazione Medici Endocrinologi medical guidelines for clinical practice for the diagnosis and management of thyroid nodules - 2016 update. Endocr Pract 22:622-639

22. Zhang M, Luo Y, Zhang Y, Tang J (2016) Efficacy and safety of ultrasound-guided radiofrequency ablation for treating low-risk papillary thyroid microcarcinoma: a prospective study. Thyroid 26(11):1581-1587

23. Wang JF, Wu T, Hu KP, Xu W, Zheng BW, Tong G et al (2017) Complications following radiofrequency ablation of benign thyroid nodules: a systematic review.Chin Med J 130(11):1361-1370 